\title{
Development and evaluation of rapid novel isothermal amplification assays for important veterinary pathogens: Chlamydia psittaci and Chlamydia pecorum
}

\author{
Martina Jelocnik ${ }^{\text {Corresp., }}{ }^{1}$, Md. Mominul Islam ${ }^{1}{ }^{\text {, Danielle Madden }}{ }^{1}$, Cheryl Jenkins ${ }^{2}$, James Branley ${ }^{3}$, Scott \\ Carver $^{4}$, Adam Polkinghorne ${ }^{1}$ \\ ${ }^{1}$ Centre for Animal Health Innovation, University of the Sunshine Coast, Maroochydore, Queensland, Australia \\ 3 Nepean Hospital, Penrith, New South Wales, Australia \\ 4 School of Biological Sciences, University of Tasmania, Hobart, Tasmania, Australia \\ Corresponding Author: Martina Jelocnik \\ Email address: Martina.Jelocnik@research.usc.edu.au
}

Background Chlamydia psittaci and Chlamydia pecorum are important veterinary pathogens, with the former also being responsible for zoonoses, and the latter adversely affecting koala populations in Australia and livestock globally. The rapid detection of these organisms is still challenging, particularly at the point-of-care (POC). In the present study, we developed and evaluated rapid, sensitive and robust $C$. psittaci-specific and C. pecorum-specific Loop Mediated Isothermal Amplification (LAMP) assays for detection of these pathogens.

Methods and materials The LAMP assays, performed in a Genie III real-time fluorometer, targeted a 263 bp region of the $C$. psittaci-specific Cps_0607 gene or a 209 bp region of a C. pecorum-specific conserved gene CpecG_0573, and were evaluated using a range of samples previously screened using species-specific quantitative PCRs (qPCRs). Species-specificity for $C$. psittaci and C. pecorum LAMP targets was tested against DNA samples from related chlamydial species and a range of other bacteria. In order to evaluate pathogen detection in clinical samples, C. psittaci LAMP was evaluated using a total of 26 DNA extracts from clinical samples from equine and avian hosts, while for $C$. pecorum LAMP, we tested a total of 63 DNA extracts from clinical samples from koala, sheep and cattle hosts. A subset of 36 C. pecorum samples was also tested in a thermal cycler (instead of a real-time fluorometer) using newly developed LAMP and results were determined as an end point detection. We also evaluated rapid swab processing (without DNA extraction) to assess the robustness of these assays.

Results Both LAMP assays were demonstrated to species-specific, highly reproducible and to be able to detect as little as 10 genome copy number/reaction, with a mean amplification time of 14 and 24 minutes for $C$. psittaci and $C$. pecorum, respectively. When testing clinical samples, the overall congruence between the newly developed LAMP assays and qPCR was $92.3 \%$ for C. psittaci $(91.7 \%$ sensitivity and $92.9 \%$ specificity); and $84.1 \%$ for $C$. pecorum (90.6 \% sensitivity and $77.4 \%$ specificity). For a subset of $36 \mathrm{C}$. pecorum samples tested in a thermal cycler using newly developed LAMP, we observed 34/36 (94.4\%) samples result being congruent between LAMP performed in fluorometer and in thermal cycler. Rapid swab processing method evaluated in this study also allows for chlamydial DNA detection using LAMP.

Discussion In this study, we describe the development of novel, rapid and robust C. psittaci-specific and C. pecorum-specific LAMP assays that are able to detect these bacteria in clinical samples in either the laboratory or POC settings. With further development and a focus on the preparation of these assays at 
the POC, it is anticipated that both tests may fill an important niche in the repertoire of ancillary diagnostic tools available to clinicians. 
1 Development and evaluation of rapid novel isothermal amplification assays for important

2

3

4 5

6

7 4558, Australia.

8 b NSW Department of Primary Industries, Elizabeth Macarthur Agricultural Institute, Menangle,

9 NSW 2568, Australia.

\#Corresponding author: Martina Jelocnik, martina.jelocnik@,research.usc.edu.au 


\section{Abstract}

18

19

20

21

22

\section{Background}

Chlamydia psittaci and Chlamydia pecorum are important veterinary pathogens, with the former also being responsible for zoonoses, and the latter adversely affecting koala populations in Australia and livestock globally. The rapid detection of these organisms is still challenging, particularly at the point-of-care (POC). In the present study, we developed and evaluated rapid, sensitive and robust C. psittaci-specific and C. pecorum-specific Loop Mediated Isothermal Amplification (LAMP) assays for detection of these pathogens.

\section{Methods and materials}

The LAMP assays, performed in a Genie III real-time fluorometer, targeted a 263 bp region of the C. psittaci-specific Cps_0607 gene or a 209 bp region of a C. pecorum-specific conserved gene CpecG_0573, and were evaluated using a range of samples previously screened using species-specific quantitative PCRs (qPCRs). Species-specificity for C. psittaci and C. pecorum LAMP targets was tested against DNA samples from related chlamydial species and a range of other bacteria.

In order to evaluate pathogen detection in clinical samples, $C$. psittaci LAMP was evaluated using a total of 26 DNA extracts from clinical samples from equine and avian hosts, while for $C$. pecorum LAMP, we tested a total of 63 DNA extracts from clinical samples from koala, sheep and cattle hosts. A subset of 36 C. pecorum samples was also tested in a thermal cycler (instead of a real-time fluorometer) using newly developed LAMP and results were determined as an end 
38 point detection. We also evaluated rapid swab processing (without DNA extraction) to assess the

39 robustness of these assays.

\section{Results}

41 Both LAMP assays were demonstrated to be species-specific, highly reproducible and able to

42 detect as little as 10 genome copy number/reaction, with a mean amplification time of 14 and 24

43 minutes for $C$. psittaci and C. pecorum, respectively. When testing clinical samples, the overall

44 congruence between the newly developed LAMP assays and qPCR was $92.3 \%$ for $C$. psittaci

45 (91.7\% sensitivity and 92.9\% specificity); and $84.1 \%$ for C. pecorum $(90.6 \%$ sensitivity and

$4677.4 \%$ specificity). For a subset of 36 C. pecorum samples tested in a thermal cycler using

47 newly developed LAMP, 34/36 (94.4\%) samples were similar positive using the fluorometer. A

48 rapid swab processing method evaluated in this study also allows for chlamydial DNA detection

49 using LAMP.

51 Discussion

52 In this study, we describe the development of novel, rapid and robust $C$. psittaci-specific and $C$.

53 pecorum-specific LAMP assays that are able to detect these bacteria in clinical samples in either

54 the laboratory or POC settings. With further development and a focus on the preparation of these

55 assays at the POC, it is anticipated that both tests may fill an important niche in the repertoire of

56 ancillary diagnostic tools available to clinicians. 
58

59

60

61

62

63

64

\section{Introduction}

The obligatory intracellular bacteria, Chlamydia psittaci and Chlamydia pecorum, are globally widespread veterinary pathogens that cause disease in an astonishing range of hosts. C. psittaci, the causative agent of psittacosis or wasting bird disease, is regarded as a major economically relevant poultry and pet bird pathogen (Knittler \& Sachse 2015; Szymanska-Czerwinska \& Niemczuk 2016). Globally, C. psittaci infections are also sporadically reported in other animal species such as pigs, cattle, sheep and horses, resulting in asymptomatic shedding, acute respiratory disease and, in the case of horses, reproductive loss (Reinhold, Sachse \& Kaltenboeck 2011; Knittler \& Sachse 2015; Jelocnik et al. 2017). Importantly, this pathogen continues to pose risks to public health through zoonotic transmission events that may lead to severe pneumonia (Gaede et al. 2008; Laroucau et al. 2015; Branley et al. 2016). This zoonotic risk is typically associated with direct contact with $C$. psittaci infected birds, although indirect contact through exposure to environmental contamination has been suggested (Branley et al. 2014; Branley et al. 2016).

C. pecorum is perhaps best known as the major pathogen of the iconic Australian native species, the koala. These infections are most commonly asymptomatic but can also result in serious inflammatory ocular and/or urogenital disease, affecting almost all Australia's mainland koala populations (Polkinghorne, Hanger \& Timms 2013; Gonzalez-Astudillo et al. 2017). C. pecorum is also an important livestock pathogen causing a range of debilitating diseases such as sporadic bovine encephalomyelitis, polyarthritis, pneumonia and conjunctivitis, with faecal shedding as a constant feature of these infections (Lenzko et al. 2011; Reinhold et al. 2011; Walker et al. 2015). In livestock, chlamydial pathogens such as C. pecorum and C. psittaci may be found as co-infections, raising the possibility of a synergistic pathogenic effect (Lenzko et al. 2011; 
81 Reinhold et al. 2011; Knittler \& Sachse 2015). The reports of chlamydial infections in novel

82 hosts and their recognised pathogenic potential (Jelocnik et al. 2015b; Burnard \& Polkinghorne

83 2016; Taylor-Brown \& Polkinghorne 2017), further highlight the need for faster detection and

84 molecular discrimination of infecting strains.

Whilst significant progress has been made in understanding the molecular epidemiology of $C$. psittaci and C. pecorum infections (Jelocnik et al. 2015a; Branley et al. 2016;), the diagnosis and detection of these pathogens is still difficult, laborious and costly, challenging efforts to manage and treat infected hosts. A variety of traditional (cell culture, antigen detection, and serology) and molecular (conventional and real-time quantitative PCR (qPCR)) diagnostic options are used to detect chlamydial infections and diagnose chlamydiosis (Sachse et al. 2009). For both $C$. psittaci and $C$. pecorum, nucleic acid amplification tests (NAATs) are presently considered the diagnostic "gold standard" due to their specificity and sensitivity, however the use of these assays is mainly restricted to research and/or diagnostic laboratories. In the absence of standardised gene target(s) for these organisms, numerous single or nested species-specific qPCR assays have been proposed and/or are used for C. psittaci (Madico et al. 2000; Geens et al. 2005; Menard et al. 2006; Branley et al. 2008) and C. pecorum (Marsh et al. 2011; Higgins et al. 2012; Wan et al. 2011; Walker et al. 2016 ) diagnosis.

The development and use of low-cost molecular diagnostic tools performed at the point-of-care (POC) which fulfil the World Health Organization “ASSURED” criteria of affordable, sensitive, specific, user-friendly, rapid, equipment-free, and deliverable to those in need to be tested, are on 
103 the exponential rise (Maffert et al. 2017). While POC testing is not necessarily required when

104 considering most chlamydial infections of veterinary concern, the ability to provide a rapid

105 detection of infections becomes of increasing significance when veterinarians and other animal

106 workers may be at risk of being exposed to $C$. psittaci infections in field or farm settings. POC

107 testing is also particularly relevant for Chlamydia screening in wild animals where laboratory

108 testing is not accessible either due to logistics associated with field sampling or that services are

109 not routinely available for testing of samples from wildlife. The latter problem is particularly

110 acute for diagnosing infections in koalas, with the recent decision to stop the production of a

111 commercially viable solid-phase ELISA leaving wildlife hospitals unable to diagnose and

112 successfully treat asymptomatic C. pecorum infections (Hanger et al. 2013).

114 While there are many options for molecular POC diagnostics, Loop Mediated Isothermal 115 Amplification (LAMP) assays developed for use in pathogen diagnostics are popular as they

116 offer significant advantages over PCR and/or serology testing (Maffert et al. 2017). Rapid,

117 simple, highly specific, easy to interpret, and carried out at a constant temperature, LAMP assays

118 can provide a diagnosis in $30 \mathrm{~min}$, in either laboratory or field setting (Mansour et al. 2015;

119 Notomi et al. 2015). Rapid isothermal LAMP assays that could be performed at the POC

120 targeting human C. pneumoniae (Kawai et al. 2009) and C. trachomatis (Jevtusevskaja et al.

121 2016; Choopara et al. 2017) infections have been proposed for use in chlamydial diagnostics.

122 Development of a C. pecorum LAMP, in particular, would meet immediate demand for koala $C$.

123 pecorum infections diagnostics, providing an alternative solution for the current laboratory

124 diagnostics. A recent outbreak of psittacosis in veterinary staff and students in contact with a $C$.

125 psittaci-infected and sick neonatal foal (Chan et al. 2017; Jelocnik et al. 2017), further 
126 demonstrates the need for POC assays such as LAMP to rapidly diagnose $C$. psittaci. In the

127 present study, we describe the development and evaluation of rapid and robust C. psittaci-

128 specific and C. pecorum-specific LAMP assays for detection of these organisms in either

129 laboratory or POC settings.

\section{Materials and methods}

Bacterial cultures and clinical samples used in this study

C. psittaci LAMP assay was evaluated using: 1) 12 DNA samples extracted from previously characterised C. psittaci isolates (10 human, two parrot and one equine) (Table S1); 2) DNA extracted from 21 placental, foetal, nasal, lung and rectal swabs, and 1 each placental and foetal tissue sample taken from 20 equine hosts; and 3) three pigeon liver DNA extracts (Table S2).

All samples were collected and submitted as part of routine diagnostic testing by field or district veterinarians to the State Veterinary Diagnostic Laboratory (SVDL), Elizabeth Macarthur Agricultural Institute (EMAI), Menangle, NSW, Australia, and as such does not require special animal ethics approval. DNA extracts from these samples were kindly provided by Dr. Cheryl Jenkins, and Dr. James Branley. The use of these swabs was considered by the University of The

142 Sunshine Coast (USC) Animal Ethics Committee and the need for further ethics consideration was waived under exemption AN/E/17/19. characterised koala $(n=7)$, sheep $(n=4)$, cattle $(n=4)$ and pig C. pecorum $(n=3)$ cultures (Table S1); 2) 16 sheep and 13 cattle ocular, rectal, and tissue swab DNA samples; and 3) 34 ocular and urogenital (UGT) koala swab DNA samples (Table S3), all available in our collection. The use 
148 of these swabs, also collected by qualified veterinarians as a part of routine diagnostic testing,

149 was considered and approved for exemption by the University of The Sunshine Coast (USC)

150 Animal Ethics Committee (AN/E/14/01 and AN/E/14/31).

152 We also evaluated the specificity of the assays against DNA samples extracted from previously 153 characterised i) chlamydial isolates (koala C. pneumoniae LPColN, C. abortus S26/3, C. suis

154 S45, C. trachomatis serovar D, C. murridarum Nigg, C. caviae GPIC) and uncultured 155 Chlamydiales (Fritschea spp.); ii) Gram negative Escherichia coli and Prevotella bivia; Gram 156 positive Fusobacterium nucleatum, Staphylococcus epidermidis, S. aureus, Streptococcus spp., 157 and Enterococcus faecalis; and iii) commercially available human gDNA (Promega, Alexandria, 158 NSW 2015), all available in our laboratory (Table S1).

In order to evaluate rapid swab processing, 18 ocular, cloacal and UGT (14 dry and four RNALater) clinical swabs taken from 14 koalas with presumptive chlamydiosis were used for testing without DNA extraction. Briefly, RNA-Later and dry swabs with added $500 \mu \mathrm{L}$ TE buffer were vortexed vigorously for $5 \min .300 \mu \mathrm{L}$ aliquots were then heated to $98^{\circ} \mathrm{C}$ for 15 min to lyse DNA, following LAMP testing. The use of these swabs, collected as a part of routine diagnostic testing, is also under Animal Ethics approval exemption (AN/E/14/01). An aliquot of $50 \mu \mathrm{L}$ of the swab suspension was used for LAMP and qPCR assays, while from the remaining volume of the swab suspension was used for DNA extraction, in order to compare swab suspension and its paired extracted DNA as a template in the assays. 
171 For the $C$. psittaci-specific LAMP gene target, we targeted a previously described conserved

172 single-copy C. psittaci-specific CDS, encoding for hypothetical protein and denoted Cpsit_0607

173 in the representative C. psittaci 6BC strain (Genbank accession number NC_015470.1) (Voigt,

174 Schöfl \& Saluz 2012). This gene was also previously proposed as a target for molecular

175 diagnosis of C. psittaci infections (Opota et al. 2015).

176 The C. pecorum-specific candidate LAMP gene target, encoding for a single-copy conserved

177 hypothetical protein and denoted CpecG_0573 in the C. pecorum MC/Marsbar koala type strain

178 (Genbank accession number NZ_CM002310.1), was selected based on a comparative genomics

179 analysis of published koala and livestock C. pecorum genomes (Jelocnik et al. 2015a). For the

180 purposes of this study, we will refer to it as Cpec_HP. Both candidate gene sequences were

181 aligned to the corresponding allele from other publicly available $C$. psittaci or $C$. pecorum strains

182 using Clustal X (as implemented in Geneious 9 (Kearse et al. 2012)), and analysed in blastn

183 against the nucleotide collection $\mathrm{nr} / \mathrm{nt}$ database to assess intra-species sequence identity, and 184 inter-species specificity.

186 For C.ps_0607 alignment, besides 6BC, we used the gene alleles from strains 84/55

187 (CP003790.1), $02 \mathrm{DC} 15$ (CP002806.1), 01DC11 (CP002805.1), WC (CP003796.1), 01DC12 and PoAn (LZRG01000000). For C. pec_HP alignment, besides MC/Marsbar, we used the gene

190 alleles from E58 (CP002608.1), P787 (CP004035.1), W73 (CP004034.1), IPA

191 (NZ_CM002311.1), NSW/Bov/SBE (NZ_JWHE00000000.1), L71 (LFRL01000000), L17 
192 (LFRK01000001), L1 (LFRH00000000), DBDeUG (NZ_CM002308.1), SA/K2/UGT

193 (SRR1693792), Nar/S22/Rec (SRR1693794) and Mer/Ovi1/Jnt (SRR1693791).

Species-specific LAMP primers were designed using the target sequences with the open-source

Primer Explorer v5 software (Eiken Chemical Co., Japan) and licensed LAMP Designer 1.15

yielded five sets of four LAMP primers including two outer (forward F3 and backward B3)

primers and two inner (forward inner FIP and backward inner BIP) primers targeting different

regions of the target gene, while LAMP Designer yielded single best set of six LAMP primers

including two outer primers (forward F3 and backward B3), two inner primers (forward inner

FIP and backward inner BIP) and two loop primers (forward loop LF and backwards loop LB).

203

204

205

All primers (as single or paired) were tested in silico, including analysing primer sequences in blast for species specificity and OligoAnalyser 3.1 (available from http://sg.idtdna.com/calc/analyzer) for primer dimerization, hairpins and melting temperatures.

After in silico and in LAMP reaction testing, a set of four primers designed by PrimerExplorer v5 and targeting a 209bp region of the $C$. pec_HP gene (spanning from position 22 to 230) was selected for C. pecorum LAMP assays performed in this study. Additional loop primers (LF/LB) were also designed to accelerate amplification time and increase sensitivity. For $C$. psittaci, a set of six primers designed with LAMP Designer and targeting a 263bp region of the C. ps_0607 gene (spanning from position 286 to 548) was selected for LAMP assays performed in this study. The specificity of primer sequences was assessed in silico using discontiquousBLAST analyses. Amplicons generated by conventional PCR using outer F3 and B3 primers for both C. psittaci and C. pecorum were gel excised, purified using Roche High Pure purification kit, and sent to 
215 Australian Genome Research Facility (AGRF) for Sanger sequencing for sequence identity 216 confirmation.

\section{$\underline{\text { LAMP assay optimisation }}$}

219 Both C. psittaci and C. pecorum LAMP assays were carried out in a $25 \mu \mathrm{L}$ reaction volume. The 220 reaction mixture consisted of $15 \mu \mathrm{L}$ Isothermal Master Mix ISO001 (Optigene, UK), $5 \mu \mathrm{L}$ six 221 primers mix (at $0.2 \mu \mathrm{M} \mathrm{F} 3$ and B3, $0.8 \mu \mathrm{M}$ FIP and BIP, and $0.4 \mu \mathrm{M}$ LF and LB) and $5 \mu \mathrm{L}$ 222 template, following LAMP assay run at $65^{\circ} \mathrm{C}$ in the Genie III real-time fluorometer (Optigene, 223 UK), as per manufacturer instructions. Following determination of the most optimal conditions 224 (fastest amplification time, fluorescence and annealing temperature), $C$. psittaci LAMP assays 225 were run at $65^{\circ} \mathrm{C}$ for $30 \mathrm{~min}$ followed by annealing step of $98-80^{\circ} \mathrm{C}$ at a rate of $0.05^{\circ} \mathrm{C} / \mathrm{s}$, while 226 C. pecorum LAMP assays were run using the same temperature and annealing conditions, 227 however for $45 \mathrm{~min}$. A negative control (LAMP mix only) was included in each run. Both $C$.

228 psittaci and $C$. pecorum LAMP assays were performed on a thermal cycle heating block at $65^{\circ} \mathrm{C}$ 229 for $30 \mathrm{~min}$, following detection of amplicons by electrophoresis on a $1.5 \%$ ethidium bromide 230 agarose gel and visualisation under UV. In addition, several C. pecorum LAMP assays were 231 conducted using the four primer set, two outer (F3 and B3) and two inner (FIP and BIP) primers, 232 on a heating block at $65^{\circ} \mathrm{C}$ for $45 \mathrm{~min}$.

234 After the assay optimisation, LAMP testing was evaluated using previously tested clinical samples, previously characterised isolates and untested new samples. C. pecorum-presumptive

236 samples were simultaneously tested using our in-house C. pecorum-specific qPCR assay (Marsh 
237 et al. 2011), while C. psittaci-presumptive samples were tested using a pan-Chlamydiales qPCR

238 assay with primers 16SIGF and 16SIGR targeting the 298bp 16S rRNA fragment (Everett, Bush

$239 \&$ Andersen 1999). Amplicon sequencing was used for the latter assay to confirm species

240 identity. The qPCR assays were carried out in a $20 \mu \mathrm{L}$ total volume, consisting of $10 \mu \mathrm{L}$

241 SYBR ${ }^{\mathrm{TM}}$ Green PCR Master Mix (Life Technologies Australia Pty Ltd., Scoresby, Vic 3179), 1

$242 \mu \mathrm{L}$ of each $10 \mu \mathrm{M}$ forward and reverse primer, $3 \mu \mathrm{L}$ miliqH2O, and $5 \mu \mathrm{L}$ DNA template. The

243 qPCR assays were run for 35 cycles $(\mathrm{Ct})$, and in each qPCR assay a positive (cultured $C$.

244 pecorum and/or C. psittaci DNA) and negative (miliqH2O) controls were included. Based on the

245 qPCR standard curve and the number of running cycles, samples amplifying at $>30 \mathrm{Ct}$ (and/or

246 equivalent detected genome copy number) were considered negative. The 23 C. psittaci-

247 presumptive equine samples were also tested with a $C$. psittaci-specific qPCR assay targeting the

248 16S rRNA gene/16S-23S rRNA spacer gene (Madico et al. 2000) at the State Veterinary

249 Diagnostic Laboratory (SVDL), Elizabeth Macarthur Agricultural Institute (EMAI), Menangle,

250 NSW, Australia. Samples amplifying at $>39 \mathrm{Ct}$ were considered negative. LAMP testing was

251 performed in a blind fashion, by two different operators, unaware of qPCR results.

252

253 Statistical analyses

254 For each assay, we compared the performance of two tests evaluated in the same population by 255 calculating Kappa and overall agreement, as well as estimated sensitivity and specificity (with 256 specified Clopper-Pearson (exact) confidence limits ) of LAMP compared to the known 257 reference (gold standard) qPCR test using EpiTools online (Sergeant 2017). It is suggested the 258 Kappa value be interpreted as follows: values $\leq 0$ as indicating no agreement and $0.01-0.20$ as 
259 none to slight, $0.21-0.40$ as fair, $0.41-0.60$ as moderate, $0.61-0.80$ as substantial, and $0.81-1.00$

260 as almost perfect agreement.

261

\section{Results and discussion}

263 With the emergence of new spill-over threats posed by C. psittaci (Laroucau et al. 2015; Jelocnik

264 et al. 2017), there is an increasing need for rapid diagnostic tools for this pathogen, particularly

265 for those that may have practical application in the field or clinical setting. There are specific

266 needs for C. pecorum POC tests as well in both the veterinary care and treatment of infected

267 domesticated and native animals, particularly in settings where veterinary diagnostic testing is

268 logistically challenging. In the present study, to the best of our knowledge, we describe the first

269 development of novel, rapid and robust C. psittaci-specific and C. pecorum-specific LAMP

270 assays that are able to detect these bacteria in clinical samples in either the laboratory or POC

271 settings.

272

273 C. psittaci and C. pecorum LAMP development

274 A C.psittaci-specific gene (C.ps_0607) was previously characterised as a conserved gene

275 sequence present only in C. psittaci genomes, and absent from all other related chlamydial

276 species (Voigt et al. 2012). BLAST analyses and alignment of the C.ps_0607 gene sequences,

277 including those from recently described human, bird and equine Australian isolates, confirmed

278 species specificity and sequence conservation. Between 0 and 13 single nucleotide

279 polymorphisms (SNPs) were observed amongst strains (100-95.1\% sequence identity) based on a

280263 bp alignment of C.ps_0607 gene sequences, including that from the most distant C. psittaci 
281 NJ1 taxon (Figure S1A). Similarly, the C. pecorum HP gene (denoted CpecG_0573 locus in

282 Marsbar strain) was determined as a highly conserved species-specific sequence following

283 BLAST analysis against publicly available sequences. Using an alignment of HP gene sequences

284 from 14 publicly available $C$. pecorum genomes, there were only 2 SNPs in the $209 \mathrm{bp}$ region to

285 be targeted by LAMP (Figure S1B).

Although multiple LAMP primer sets were predicted, LAMP primer sets denoted in Figure 1 were chosen for further assay development. For C. psittaci assays, a set designed using LAMP Explorer was utilised while, for C. pecorum, we used a set designed with PrimerExplorer (Table 1). After initial testing, some of the predicted primer sets were discarded due to i) potential crossamplification associated with a lack of specificity of the target primer; (ii) not achieving an amplification signal in the fluorometer; and iii) amplifying non-specific targets, including positive amplification in negative controls (data not shown). While we achieved initial amplification of a $C$. psittaci single copy dilution in a $30 \mathrm{~min}$ assay using the designed LAMP primer set, initial reaction times for a $C$. pecorum single copy amplification averaged $50 \mathrm{~min}$. In order to accelerate amplification times for C. pecorum, we additionally designed a pair of Loop primers for the $C$. pecorum set which decreased the amplification of a single copy to $30 \mathrm{~min}$. LAMP assays using DNA extracts from 12 C. psittaci and 18 C. pecorum cultured isolates, DNA extracts from other chlamydial species and a range of DNA extracts from other bacteria. Positive amplification as assessed by the presence of an observable amplification curve characterised by a 
303 specific melt was observed only for the target species in their respective assays (Table S1). No 304 amplification curves were observed for any of the non-targeted chlamydial species or other 305 bacteria included in our specificity assays (Table S1). The C. pecorum and C. psittaci LAMP 306 assays did not amplify either the related chlamydial species or other bacteria included in our 307 specificity assays. In this study, in contrast, a previously described "C. pecorum-specific” qPCR 308 assay (Marsh et al. 2011; Wan et al. 2011) showed positive amplification and melt for $C$. 309 psittaci and C. pneumoniae DNA samples.

310 The choice to use the C.ps_0607 gene as a LAMP target was straight forward since it had been 311 suggested for such a purpose in previous studies (Voigt et al. 2012; Opota et al. 2015), For $C$. 312 pecorum, however, we utilised our ongoing comparative genomics to select $C$. pecorum-specific 313 and conserved C.pec_HP gene described in this study for the first time. In silico analyses and 314 assay development confirmed species-specificity of this gene and its suitability for use in 315 diagnostic assays. Previously published $C$. pecorum diagnostic assays targeted highly polymorphic genes such as ompA (Higgins et al. 2012; Yang et al. 2014), which may require the use of probes due to sequence variation, prolonging the detection time and increasing diagnostic costs. Our routinely used in house C. pecorum-specific assay which targets a 204 bp 16S rRNA fragment (Marsh et al. 2011; Wan et al. 2011) was simpler to use, however we have shown that this assay may cross-react with other related chlamydial species due to a lack of sufficient sequence variation in the region of the 16S rRNA gene targeted (Bachmann, Polkinghorne \&

322 Timms 2014). For koala diagnostics where C. pecorum is the most abundant and prevalent chlamydial organism (Polkinghorne et al. 2013), this cross-reactivity may not be of a big concern. For the veterinary diagnosis of infections in livestock where co-infections with several chlamydial species are common (Lenzko et al. 2011; Reinhold et al. 2011), this assay may be 
326 less suitable. Using the $C$. pecorum-specific HP gene as a target in different diagnostic assays

327 would hence seem promising.

329 Performance of the C. psittaci and C. pecorum LAMP assays

330 The sensitivity of the LAMP assays was evaluated using $5 \mu \mathrm{L}$ cultured $C$. psittaci and $C$.

331 pecorum gDNA in 10-fold serial dilutions as a template in assays performed in triplicate in

332 separate runs. The limits of detection of the LAMP assays were conservatively 10 copies for $C$.

333 psittaci, with $3 / 3(100 \%)$ positive amplification for 10 copy dilutions for $C$. psittaci, and 1 copy

334 for $C$. pecorum, with $3 / 3(100 \%)$ positive amplifications for a single copy dilution of $C$. pecorum

335 DNA (Table 2 and 3). In the final and optimised LAMP assays, the mean amplification time

336 detecting the lower limit (a single copy) for $C$. psittaci was 14.23 min with an average $84.45^{\circ} \mathrm{C}$

337 melt (Table 2) while, for C. pecorum, it was 24 min with an average $83.42^{\circ} \mathrm{C}$ melt (Table 3 ).

338 Comparing the two newly developed assays, $C$. psittaci LAMP had the faster run time than that

339 of $C$. pecorum LAMP. This difference in assays kinetics could be attributed to the improved $C$.

340 psittaci LAMP primers design, as they were predicted by the LAMP Designer software

341 (Nagamine, Hase \& Notomi 2002). As we additionally designed Loop primers for C. pecorum,

342 we can anticipate an improvement in the $C$. pecorum assay kinetics by re-designing the loop

343 primers (e.g. extending the sequence to $20-22 \mathrm{bp}$ ), as well as testing LAMP mixes in different

344 ratios and with improved polymerases.

346 In order to test the reproducibility of our LAMP assays, we tested a subset of C. pecorum and $C$.

347 psittaci PCR positive samples (Table 4). All samples were run in a "blind fashion", in triplicate 
348 and in separate runs by two different operators. For both assays, the amplification times and

349 melts of each sample between the runs were very similar, with 0 to $1.5 \mathrm{~min}$ (SDs ranging from 0

$350-0.98$ ) difference in amplification times for each sample, and 0.03 to $0.83^{\circ} \mathrm{C}$ (SDs ranging from

$3510.02-0.26)$ difference in melt for each sample. Congruence between the runs performed by

352 different operators indicates that both LAMP assays described in this study are highly

353 reproducible, and can detect the target organism in less than 30 min even when in low infectious

354 loads of $<10$ copies.

$\underline{\text { Pathogen detection in clinical samples using newly developed LAMP }}$

357 For C. psittaci, a total of 27 DNA extracts from clinical samples were tested with both C. psittaci

358 LAMP and qPCR assays (Table S2). For these analyses, samples with $>20$ min amplification

359 time were considered negative for LAMP, while for qPCR, samples with $<20$ genome

360 copy/reaction and/or $>30 \mathrm{Ct}$ (quantification cycle) were considered negative, based on the qPCR

361 standard curve and the number of running cycles used for this testing. As observed in Table S2

362 and based on above cut-off values, 24/26 (92.3\%) samples were congruent between the two tests,

363 with 11 samples positive and 13 samples negative by both (Table 5). For 2/26 (7.7\%) where

364 there was disagreement, one sample was LAMP positive but qPCR negative, and another was

365 qPCR positive but C. psittaci LAMP negative. Based on these results, the Kappa value was

366 calculated at $0.85(95 \%$ CI $0.64-1.05)$ indicating an almost perfect agreement between the tests.

367 The overall sensitivity of the $C$. psittaci LAMP was 91.7\% (Clopper-Pearson 95\% CI 0.62 -

368 0.99) and with 92.9\% (Clopper-Pearson 95\% CI $0.66-0.99$ ) specificity, compared to the qPCR

369 used in this study. In addition, a subset of 23 samples was also tested independently by a third 
370 party. Using a cut off of $>\mathrm{Ct} 39$ as negative, 19/23 (82.60\%) of these test results were in

371 congruence with our C. psittaci LAMP results (Table S2).

373 For C. pecorum, we tested a total of 63 DNA extracts from clinical samples from several animal

374 hosts by both LAMP and qPCR (Table S3). For these analyses, samples with $>30$ min

375 amplification time were considered negative for LAMP, while for qPCR, samples with $<35$

376 genome copy /reaction and/or $>30 \mathrm{Ct}$ were considered negative based on the standard curve and

377 number of run cycles used for this testing. For the 63 clinical samples, the overall congruence

378 was $84.1 \%$ with a Kappa value of 0.68 (95\% CI $0.50-0.86)$, indicating substantial agreement

379 between the tests. Congruent results between tests were obtained for 53 samples, while there

380 were 10 discrepant samples using the above cut off for C. pecorum (Table 6). The overall

381 sensitivity of $C$. pecorum LAMP was 90.6\% (Clopper-Pearson 95\% CI $0.75-0.98$ ), while

382 specificity was $77.4 \%$ (Clopper-Pearson 95\% CI $0.59-0.90$ ) in comparison to the qPCR assay.

383 A subset of 36 C pecorum samples was also tested in a thermal cycler using the newly

384 developed LAMP and results were determined as an end point detection. For this experiment,

$38534 / 36(94.4 \%)$ samples were congruent between LAMP performed in fluorometer and in a

386 thermal cycler (Table S3), demonstrating the robustness of the C. pecorum LAMP (Figure S2).

388 Considering that the qPCR assay used in this study to quantify and detect $C$. psittaci is

389 chlamydial genus rather species specific (Everett et al. 1999), high congruence observed for $C$.

390 psittaci assays could be attributed to testing a limited set of samples taken from hosts with

391 presumptive $C$. psittaci chlamydiosis. Lower congruence between the $C$. pecorum-specific 
392 assays could be due to technical and experimental aspects and characteristics (such as the assay

393 efficiency, analytical sensitivity, template preparation) (Bustin et al. 2010) of the C. pecorum

$39416 \mathrm{~S}$ qPCR assay used in this study. As a sidenote, we also evaluated the use of C. psittaci and $C$.

395 pecorum LAMP targets (263bp of the C. ps_0607 and 209bp C. pec_HP genes, respectively)

396 using outer F3 and B3 primers in a fluorescence-based (SybrGreen) qPCR assays, if needed to

397 estimate infectious loads of the pathogen. In this preliminary analyses, both targets seem suitable

398 for use in qPCR assays as well, as we were able to detect low infectious load up to 10

399 copies/reaction in a sample.

400

$401 \quad$ Rapid swab processing

402 Rapid swab processing and using the swab suspension directly in LAMP assays were previously 403 successfully evaluated for testing for respiratory syncytial virus from nasopharyngeal swabs

404 (Mahony et al. 2013) and rapid detection of Streptococcus agalactiae in vaginal swabs

405 (McKenna et al. 2017). A recent study also demonstrated that C. trachomatis can be detected

406 directly from urine samples using the LAMP method (Jevtusevskaja et al. 2016). In this study,

407 we also evaluated rapid swab processing without DNA extraction in order to begin to assess the

408 POC potential of these assays. A total of 18 swabs taken from conjunctival and urogenital sites

409 from koalas with presumptive chlamydiosis, of which four were stored in RNA Later and 14

410 were dry, were used for this experiment (Table 7). Vigorously vortexed and heated swab

411 suspension samples were directly used as a template in both C. pecorum LAMP reaction

412 performed in fluorometer and qPCR assay. We also performed DNA extraction from the swabs

413 to be used as a comparison to rapid swab processing. We did not detect C. pecorum DNA in any

414 of the RNA Later suspensions either by LAMP nor qPCR assay (Table 7), in contrast to 
415 detecting C. pecorum in 50\% (2/4) of the DNA extracts from the swabs by both methods. Using

416 the rapidly processed swab suspension as a template, C. pecorum was detected in $6 / 14$ by

417 LAMP, and only 2/14 by qPCR (Table 7). The swab suspension LAMP results were 92.8\%

$418(13 / 14)$ congruent to the LAMP results and 85.7\% congruent (12/14) to the qPCR results using

419 the swab's paired DNA sample. In order to evaluate the potential presence of inhibitors in our

420 samples, we "spiked" negative swab suspensions and its paired DNA samples with known

421 amounts of $C$. pecorum $\left(1 \times 10^{\wedge} 4\right.$ copies/reaction). As observed in Table 7 , we detected $C$.

422 pecorum by both LAMP and qPCR in "spiked" negative samples derived from dry swabs. No $C$.

423 pecorum was detected in "spiked" RNA Later swab suspension, indicating the potential presence

424 of inhibitors in these reactions. Our results suggest that the LAMP assays are capable of

425 amplifying specific amplification products from crude DNA extracts.

427 Further work is additionally required to enhance the POC capabilities of these new chlamydial

428 LAMP assays to meet the clinical need including (i) the evaluation of rapid swab processing 429 methods using commercially available DNA release portable devices and/or sample preparation 430 using microfluidic support; (ii) alternative amplification detection methods such as visible 431 colorimetric or turbidimetric change and/or solid-phase 'dipstick' tests (Maffert et al. 2017).

432 With further development and the aforementioned focus on the preparation of these assays at the 433 POC (Parida et al. 2008; Tomita et al. 2008), it is anticipated that both LAMP tests described in 434 this study may fill an important niche in the repertoire of ancillary diagnostic tools available to 435 clinicians. 


\section{Acknowledgments}

438 We thank Prof. James Mahony, Dr. Catherine Chicken, Dr. Joan Carrick, Dr. Ian Marsh, Narelle 439 Sales and Dr. Bill Lott for their helpful advice on POC assays. We also thank Dr. Brendon

440 O’Rourke, Sankhya Bommana, Sharon Nyari, Noa Ziklo and Alyce Taylor-Brown for provision 441 of DNA samples used in this study. 
442

443

444

445

446

447

448

449

450

451

452

453

454

455

456

457

458

459

460

461

462

463

464

465

466

467

468

469

470

471

472

\section{References}

Bachmann NL, Polkinghorne A, Timms P. 2014. Chlamydia genomics: providing novel insights into chlamydial biology. Trends in Microbiology 22:464-472. DOI:10.1016/j.tim.2014.04.013

Branley J, Bachmann NL, Jelocnik M, Myers GS, Polkinghorne A. 2016. Australian human and parrot Chlamydia psittaci strains cluster within the highly virulent $6 \mathrm{BC}$ clade of this important zoonotic pathogen. Scientific Reports 6:30019. DOI:10.1038/srep30019

Branley JM, Roy B, Dwyer DE, Sorrell TC. 2008. Real-time PCR detection and quantitation of Chlamydophila psittaci in human and avian specimens from a veterinary clinic cluster. European Journal of Clinical Microbiology and Infectious Diseases 27:269-273. DOI:10.1007/s10096-007-0431-0

Branley JM, Weston KM, England J, Dwyer DE, Sorrell TC. 2014. Clinical features of endemic community-acquired psittacosis. New Microbes New Infections 2:7-12. DOI:10.1002/2052-2975.29

Burnard D, and Polkinghorne A. 2016. Chlamydial infections in wildlife-conservation threats and/or reservoirs of 'spill-over' infections? Veterinary Microbiology 196:78-84. http://dx.doi.org/10.1016/j.vetmic.2016.10.018

Bustin SA, Beaulieu JF, Huggett J, Jaggi R, Kibenge FS, Olsvik PA, Penning LC, Toegel S. 2010. MIQE precis: Practical implementation of minimum standard guidelines for fluorescence-based quantitative real-time PCR experiments. BMC Molecular Biology 11:74. DOI:10.1186/1471-2199-11-74

Chan J, Doyle B, Branley J, Sheppeard V, Gabor M, Viney K, Quinn H, Janover O, McCready M, Heller J. 2017. An outbreak of psittacosis at a veterinary school demonstrating a novel source of infection. One Health 3:29-33.

Choopara I, Arunrut N, Kiatpathomchai W, Dean D, Somboonna N. 2017. Rapid and visual Chlamydia trachomatis detection using loop-mediated isothermal amplification and hydroxynaphthol blue. Letters in Applied Microbiology 64:51-56. DOI:10.1111/lam.12675

Everett KDE, Bush RM, Andersen AA. 1999. Emended description of the order Chlamydiales, proposal of Parachlamydiaceae fam. nov. and Simkaniaceae fam. nov., each containing one monotypic genus, revised taxonomy of the family Chlamydiaceae, including a new 
473

474

475

476

477

478

479

480

481

482

483

484

485

486

487

488

489

490

491

492

493

494

495

496

497

498

499

500

501

502

genus and five new species, and standards for the identification of organisms.

International Journal of Systematic Bacteriology 49:415-440. DOI:10.1099/00207713$49-2-415$

Gaede W, Reckling KF, Dresenkamp B, Kenklies S, Schubert E, Noack U, Irmscher HM, Ludwig C, Hotzel H, Sachse K. 2008. Chlamydophila psittaci infections in humans during an outbreak of psittacosis from poultry in Germany. Zoonoses Public Health 55:184-188. DOI:10.1111/j.1863-2378.2008.01108.x

Geens T, Dewitte A, Boon N, Vanrompay D. 2005. Development of a Chlamydophila psittaci species-specific and genotype-specific real-time PCR. Veterinary Research 36:787-797.

Gonzalez-Astudillo V, Allavena R, McKinnon A, Larkin R, Henning J. 2017. Decline causes of Koalas in South East Queensland, Australia: a 17-year retrospective study of mortality and morbidity. Scientific Reports 7:42587. DOI:10.1038/srep42587

Hanger J, Loader J, Wan C, Beagley KW, Timms P, Polkinghorne A. 2013. Comparison of antigen detection and quantitative PCR in the detection of chlamydial infection in koalas (Phascolarctos cinereus). The Veterinary Journal 195:391-393. http://dx.doi.org/10.1016/j.tvj1.2012.07.024

Higgins DP, Beninati T, Meek M, Irish J, Griffith JE. 2012. Within-population diversity of koala Chlamydophila pecorum at ompA VD1-VD3 and the ORF663 hypothetical gene. Veterinary Microbiology 156:353-358. 10.1016/j.vetmic.2011.11.005

Jelocnik M, Bachmann NL, Kaltenboeck B, Waugh C, Woolford L, Speight KN, Gillett A, Higgins DP, Flanagan C, Myers GS, Timms P, Polkinghorne A. 2015a. Genetic diversity in the plasticity zone and the presence of the chlamydial plasmid differentiates Chlamydia pecorum strains from pigs, sheep, cattle, and koalas. BMC Genomics 16:893. DOI:10.1186/s12864-015-2053-8

Jelocnik M, Branley J, Heller J, Raidal S, Alderson S, Galea F, Gabor M, Polkinghorne A. 2017. Multilocus sequence typing identifies an avian-like Chlamydia psittaci strain involved in equine placentitis and associated with subsequent human psittacosis. Emerging Microbes and Infection 6:e7. DOI:10.1038/emi.2016.135

Jelocnik M, Self R, Timms P, Borel N, Polkinghorne A. 2015b. Novel sequence types of Chlamydia pecorum infect free-ranging Alpine ibex (Capra ibex) and red deer (Cervus 
503

504

505

506

507

508

509

510

511

512

513

514

515

516

517

518

519

520

521

522

523

524

525

526

527

528

529

530

531

elaphus) in Switzerland. Journal of Wildlife Diseases 51:479-483. DOI:10.7589/2014-08220

Jevtusevskaja J, Uusna J, Andresen L, Krolov K, Laanpere M, Grellier T, Tulp I, Langel U. 2016. Combination with antimicrobial peptide lyses improves loop-mediated isothermal amplification based method for Chlamydia trachomatis detection directly in urine sample. BMC Infectious Diseases 16:329. DOI:10.1186/s12879-016-1674-0

Kawai Y, Miyashita N, Kishi F, Tabuchi M, Oda K, Yamaguchi T, Kawasaki K, Yamazaki T, Ouchi K. 2009. Development and evaluation of a loop-mediated isothermal amplification method for the rapid detection of Chlamydophila pneumoniae. European Journal of Clinical Microbiology and Infectious Diseases 28:801-805. DOI:10.1007/s10096-0090710-z

Kearse M, Moir R, Wilson A, Stones-Havas S, Cheung M, Sturrock S, Buxton S, Cooper A, Markowitz S, Duran C, Thierer T, Ashton B, Meintjes P, Drummond A. 2012. Geneious Basic: an integrated and extendable desktop software platform for the organization and analysis of sequence data. Bioinformatics 28:1647-1649.

DOI:10.1093/bioinformatics/bts199

Knittler MR, and Sachse K. 2015. Chlamydia psittaci: update on an underestimated zoonotic agent. Pathogens and Disease 73:1-15. DOI:10.1093/femspd/ftu007

Laroucau K, Aaziz R, Meurice L, Servas V, Chossat I, Royer H, de Barbeyrac B, Vaillant V, Moyen JL, Meziani F, Sachse K, Rolland P. 2015. Outbreak of psittacosis in a group of women exposed to Chlamydia psittaci-infected chickens. Euro Surveillance 20:pii $=21155$.

Lenzko H, Moog U, Henning K, Lederbach R, Diller R, Menge C, Sachse K, Sprague L. 2011. High frequency of chlamydial co-infections in clinically healthy sheep flocks. $B M C$ Veterinary Research 7:29. DOI:10.1186/1746-6148-7-29

Madico G, Quinn TC, Boman J, Gaydos CA. 2000. Touchdown enzyme time release-PCR for detection and identification of Chlamydia trachomatis, C. pneumoniae, and C. psittaci using the 16S and 16S-23S spacer rRNA genes. Journal of Clinical Microbiology 38:1085-1093. 
532 Maffert P, Reverchon S, Nasser W, Rozand C, and Abaibou H. 2017. New nucleic acid testing

533 devices to diagnose infectious diseases in resource-limited settings. European Journal of

534 Clinical Microbiology and Infectious Diseases. DOI:10.1007/s10096-017-3013-9

535 Mahony J, Chong S, Bulir D, Ruyter A, Mwawasi K, Waltho D. 2013. Development of a

536 sensitive loop-mediated isothermal amplification assay that provides specimen-to-result

537 diagnosis of respiratory syncytial virus infection in 30 minutes. Journal of Clinical

538 Microbiology 51:2696-2701. DOI:10.1128/jcm.00662-13

539 Mansour SM, Ali H, Chase CC, and Cepica A. 2015. Loop-mediated isothermal amplification

540 for diagnosis of 18 World Organization for Animal Health (OIE) notifiable viral diseases

541 of ruminants, swine and poultry. Animal Health Research Reviews 16:89-106.

$542 \quad$ DOI: $10.1017 / \mathrm{s} 1466252315000018$

543 Marsh J, Kollipara A, Timms P, Polkinghorne A. 2011. Novel molecular markers of Chlamydia 544 pecorum genetic diversity in the koala (Phascolarctos cinereus). BMC Microbiology

545 11:77. DOI:10.1186/1471-2180-11-77

546 McKenna JP, Cox C, Fairley DJ, Burke R, Shields MD, Watt A, Coyle PV. 2017. Loop547 mediated isothermal amplification assay for rapid detection of Streptococcus agalactiae (group B streptococcus) in vaginal swabs - a proof of concept study. Journal of Medical Microbiology 66:294-300. DOI:10.1099/jmm.0.000437

Menard A, Clerc M, Subtil A, Megraud F, Bebear C, de Barbeyrac B. 2006. Development of a real-time PCR for the detection of Chlamydia psittaci. Journal of Medical Microbiology 55:471-473. DOI:10.1099/jmm.0.46335-0

Nagamine K, Hase T, Notomi T. 2002. Accelerated reaction by loop-mediated isothermal amplification using loop primers. Molecular and Cellular Probes 16:223-229.

Notomi T, Mori Y, Tomita N, Kanda H. 2015. Loop-mediated isothermal amplification (LAMP): principle, features, and future prospects. Journal of Microbiology 53:1-5. DOI:10.1007/s12275-015-4656-9

Opota O, Jaton K, Branley J, Vanrompay D, Erard V, Borel N, Longbottom D, Greub G. 2015. Improving the molecular diagnosis of Chlamydia psittaci and Chlamydia abortus infection with a species-specific duplex real-time PCR. Journal of Medical Microbiology 64:1174-1185. DOI:10.1099/jmm.0.000139 
562 Parida M, Sannarangaiah S, Dash PK, Rao PV, Morita K. 2008. Loop mediated isothermal 563 amplification (LAMP): a new generation of innovative gene amplification technique; 564 perspectives in clinical diagnosis of infectious diseases. Reviews in Medical Virology 565

566 567 568 569 570 571 18:407-421. DOI:10.1002/rmv.593

Polkinghorne A, Hanger J, Timms P. 2013. Recent advances in understanding the biology, epidemiology and control of chlamydial infections in koalas. Veterinary Microbiology 165:214-223. DOI:10.1016/j.vetmic.2013.02.026

Reinhold P, Sachse K, Kaltenboeck B. 2011. Chlamydiaceae in cattle: Commensals, trigger organisms, or pathogens? The Veterinary Journal 189:257-267. DOI:10.1016/j.tvj1.2010.09.003

Sachse K, Vretou E, Livingstone M, Borel N, Pospischil A, Longbottom D. 2009. Recent developments in the laboratory diagnosis of chlamydial infections. Veterinary Microbiology 135:2-21. http://dx.doi.org/10.1016/j.vetmic.2008.09.040

Sergeant ESG. 2017. Epitools epidemiological calculators. Available at http://epitools.ausvet.com.au

Szymanska-Czerwinska M, and Niemczuk K. 2016. Avian Chlamydiosis zoonotic disease. Vector Borne and Zoonotic Diseases 16:1-3. DOI:10.1089/vbz.2015.1839

Taylor-Brown A, and Polkinghorne A. 2017. New and emerging chlamydial infections of creatures great and small. New Microbes New Infections 18:28-33. DOI:10.1016/j.nmni.2017.04.004

Tomita N, Mori Y, Kanda H, Notomi T. 2008. Loop-mediated isothermal amplification (LAMP) of gene sequences and simple visual detection of products. Nature Protocols 3:877-882. DOI:10.1038/nprot.2008.57

Voigt A, Schöfl G, Saluz HP. 2012. The Chlamydia psittaci Genome: A comparative analysis of intracellular pathogens. PLoS ONE 7:e35097. DOI:10.1371/journal.pone.0035097

Walker E, Lee EJ, Timms P, Polkinghorne A. 2015. Chlamydia pecorum infections in sheep and cattle: A common and under-recognised infectious disease with significant impact on animal health. Veterinary Journal 206:252-260. DOI:10.1016/j.tvj1.2015.09.022

Walker E, Moore C, Shearer P, Jelocnik M, Bommana S, Timms P, Polkinghorne A. 2016. Clinical, diagnostic and pathologic features of presumptive cases of Chlamydia pecorum- 
592 associated arthritis in Australian sheep flocks. BMC Veterinary Research 12:193.

593 DOI:10.1186/s12917-016-0832-3

594 Wan C, Loader J, Hanger J, Beagley KW, Timms P, Polkinghorne A. 2011. Using quantitative 595 polymerase chain reaction to correlate Chlamydia pecorum infectious load with ocular, 596 urinary and reproductive tract disease in the koala (Phascolarctos cinereus). Australian 597 Veterinary Journal 89:409-412. DOI:10.1111/j.1751-0813.2011.00827.x

598 Yang R, Jacobson C, Gardner G, Carmichael I, Campbell AJ, Ryan U. 2014. Longitudinal 599 prevalence and faecal shedding of Chlamydia pecorum in sheep. Veterinary Journal $600 \quad$ 201:322-326. DOI:10.1016/j.tvj1.2014.05.037 


\section{Figure 1}

LAMP primer sequences and positions in the target gene regions.

A) C. psittaci LAMP primer set; and B) C. pecorum LAMP primer set. Outer F3 and B3 primers are indicated in green, inner FIP and BIP in blue, and loop LF and BL in pink colour.

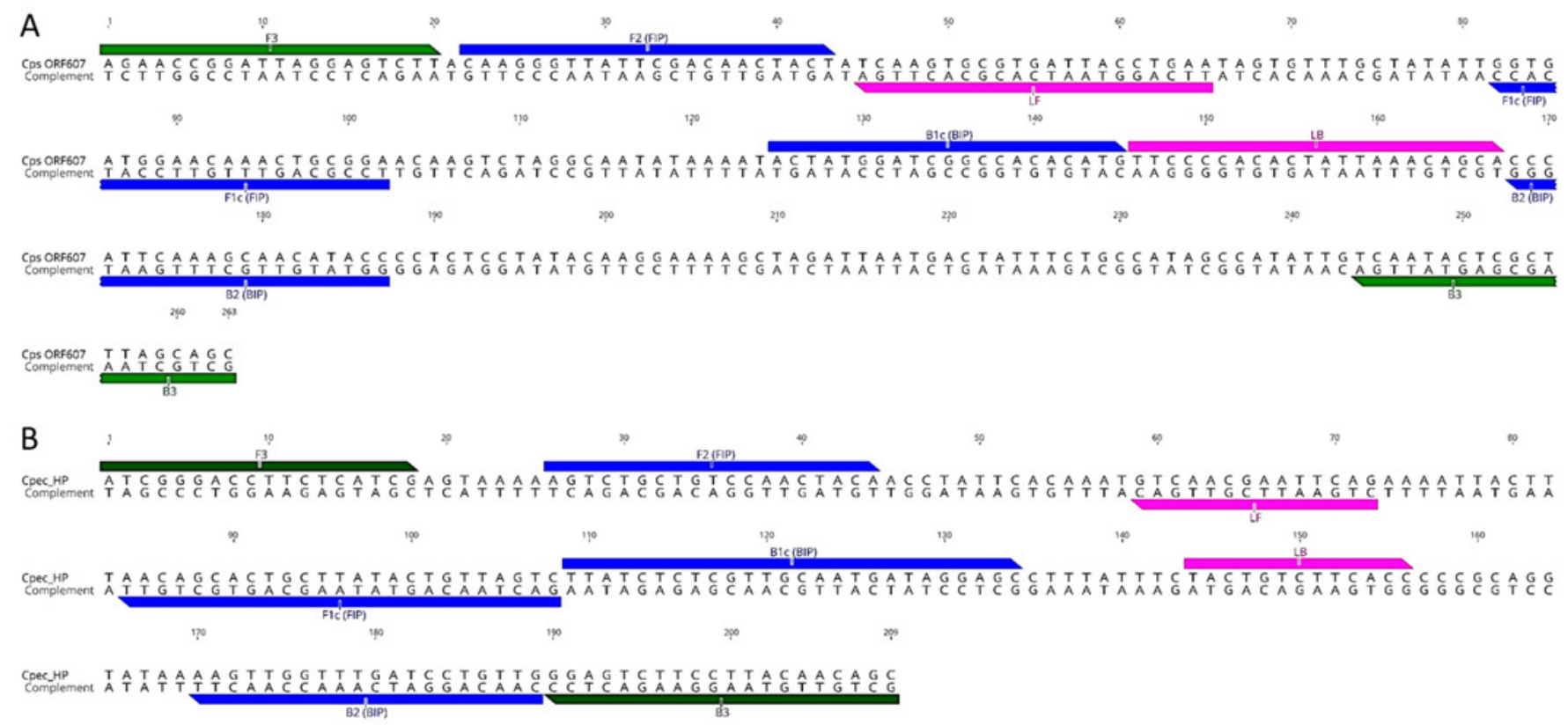


Table $\mathbf{1}$ (on next page)

LAMP primers set used in this study. 
1 Table 1. LAMP primers set used in this study.

\begin{tabular}{|c|c|c|c|}
\hline \multicolumn{4}{|c|}{ C. psittaci LAMP primers } \\
\hline Name & Sequence 5' - 3' & $\begin{array}{c}\text { Positio } \\
\text { n }\end{array}$ & $\begin{array}{c}\text { Lengt } \\
\text { h }\end{array}$ \\
\hline F3 & AGAACCGGATTAGGAGTCTT & 286 & 20 \\
\hline B3 & GCTGCTAAAGCGAGTATTGA & 548 & 20 \\
\hline $\begin{array}{c}\mathrm{FIP}(\mathrm{F} 1 \mathrm{c}+\mathrm{F} 2 \\
\quad)\end{array}$ & $\begin{array}{l}\text { TCCGCAGTTTGTTCCATCACCCAAGGGTTATTCGACAACTA } \\
\text { CT }\end{array}$ & & 43 \\
\hline $\begin{array}{l}\mathrm{BIP}(\mathrm{B} 1 \mathrm{c}+\mathrm{B} \\
2)\end{array}$ & ACTATGGATCGGCCACACATGGGTATGTTGCTTTGAATGGG & & 41 \\
\hline LoopF & TTCAGGTAATCACGCACTTGA & 350 & 21 \\
\hline LoopB & TTCCCCACACTATTAAACAGCA & 431 & 22 \\
\hline F2 & CAAGGGTTATTCGACAACTACT & 307 & 22 \\
\hline F1c & TCCGCAGTTTGTTCCATCACC & 387 & 21 \\
\hline $\mathrm{B} 2$ & GGTATGTTGCTTTGAATGGG & 472 & 20 \\
\hline $\mathrm{B} 1 \mathrm{c}$ & ACTATGGATCGGCCACACATG & 410 & 21 \\
\hline \multicolumn{4}{|c|}{ C. pecorum LAMP primers } \\
\hline Name & Sequence 5' - 3' & $\begin{array}{c}\text { Positio } \\
\text { n }\end{array}$ & $\begin{array}{c}\text { Lengt } \\
\text { h }\end{array}$ \\
\hline F3 & ATCGGGACCTTCTCATCG & 22 & 18 \\
\hline B3 & GCTGTTGTAAGGAAGACTCC & 230 & 20 \\
\hline $\begin{array}{c}\mathrm{FIP}(\mathrm{F} 1 \mathrm{c}+\mathrm{F} 2 \\
\quad)\end{array}$ & $\begin{array}{l}\text { GACTAACAGTATAAGCAGTGCTGTTAGTCTGCTGTCCAACT } \\
\text { ACA }\end{array}$ & & 44 \\
\hline $\begin{array}{l}\mathrm{BIP}(\mathrm{B} 1 \mathrm{c}+\mathrm{B} \\
2) \\
\end{array}$ & $\begin{array}{l}\text { TTATCTCTCGTTGCAATGATAGGAGCCAACAGGATCAAACC } \\
\text { AACTT }\end{array}$ & & 46 \\
\hline LoopF & CTGAATTCGTTGAC & 93 & 14 \\
\hline LoopB & TACTGTCTTCACC & 165 & 12 \\
\hline F2 & AGTCTGCTGTCCAACTACA & 47 & 19 \\
\hline F1c & GACTAACAGTATAAGCAGTGCTGTT & 129 & 25 \\
\hline $\mathrm{B} 2$ & CAACAGGATCAAACCAACTT & 210 & 20 \\
\hline $\mathrm{B} 1 \mathrm{c}$ & TTATCTCTCGTTGCAATGATAGGAGC & 130 & 26 \\
\hline
\end{tabular}




\section{Table 2 (on next page)}

C. psittaci LAMP assaya sensitivity. 
1 Table 2. C. psittaci LAMP assay a sensitivity.

\begin{tabular}{|c|c|c|c|c|}
\hline Dilution* & $\begin{array}{c}\text { Time to } \\
\text { amplify }(\min )\end{array}$ & $\begin{array}{l}\text { Melt } \\
\left({ }^{\circ} \mathrm{C}\right)\end{array}$ & $\begin{array}{c}\text { Time } \\
\text { (Mean }+ \\
\text { SD) }\end{array}$ & $\begin{array}{c}\text { Melt } \\
\text { (Mean + } \\
\text { SD) }\end{array}$ \\
\hline $10^{\wedge} 6$ & 5.15 & 84.43 & & \\
\hline $10^{\wedge} 6$ & 5.00 & 84.46 & $5.10,0.09$ & $84.49,0.08$ \\
\hline $10^{\wedge} 6$ & 5.15 & 84.58 & & \\
\hline $10^{\wedge} 5$ & 6.30 & 84.34 & & \\
\hline $10^{\wedge} 5$ & 6.45 & 84.33 & $6.30,0.15$ & $84.37,0.06$ \\
\hline $10^{\wedge} 5$ & 6.15 & 84.43 & & \\
\hline $10^{\wedge} 4$ & 7.15 & 84.59 & & \\
\hline $10^{\wedge} 4$ & 7.30 & 84.58 & $7.25,0.09$ & $84.56,0.04$ \\
\hline $10^{\wedge} 4$ & 7.30 & 84.51 & & \\
\hline $10^{\wedge} 3$ & 8.45 & 84.46 & & \\
\hline $10^{\wedge} 3$ & 8.15 & 84.43 & $8.25,0.173$ & $84.44,0.01$ \\
\hline $10^{\wedge} 3$ & 8.15 & 84.44 & & \\
\hline 100 & 9.15 & 84.48 & & \\
\hline 100 & 9.30 & 84.39 & $9.30,0.15$ & $84.46,0.06$ \\
\hline 100 & 9.45 & 84.51 & & \\
\hline 10 & 12.00 & 84.41 & & \\
\hline 10 & 11.00 & 84.35 & $11.33,0.58$ & $84.38,0.03$ \\
\hline 10 & 11.00 & 84.39 & & \\
\hline 1 & 16.00 & 84.44 & & \\
\hline 1 & 0.00 & 0 & $14.23,2.51$ & $84.34,0.14$ \\
\hline 1 & 12.45 & 84.24 & & \\
\hline 0.1 & 25.25 & 84.20 & & \\
\hline 0.1 & $-b$ & - & - & 84.20 \\
\hline 0.1 & - & $84.20^{c}$ & & \\
\hline
\end{tabular}

2 a The assay was performed in Genie III Real-time fluorometer, with the amplification times and annealing 3 temperatures recorded at the end of each run. The samples were tested in three different runs; *Template 4 was serially diluted $C$. psittaci CR009 gDNA which genome copy number was determined by qPCR. ${ }^{\text {b: }}$

5 No amplification detected; ${ }^{\text {: }}$ No amplification, but melt and annealing curve recorded. 


\section{Table 3(on next page)}

C. pecorum LAMP assay" sensitivity.

\# The assay was performed in Genie III Real-time fluorometer, with the amplification times and annealing temperatures recorded at the end of each run. The samples were tested in different runs; *Template was serially diluted $C$. pecorum gDNA which genome copy number

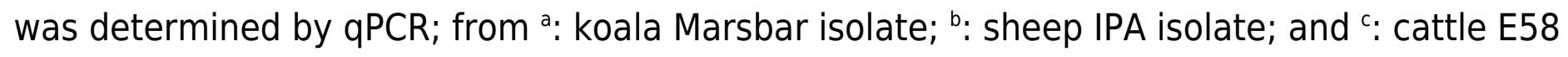
isolate; ${ }^{\text {: }}$ No amplification, but melt and annealing curve recorded. 
1 Table 3. C. pecorum LAMP assay" sensitivity.

\begin{tabular}{ccccc}
\hline Dilution* & $\begin{array}{c}\text { Time to } \\
\text { amplify }(\mathrm{min})\end{array}$ & $\begin{array}{c}\text { Melt } \\
\left({ }^{\circ} \mathrm{C}\right)\end{array}$ & $\begin{array}{c}\text { Time } \\
(\text { Mean }+ \\
\text { SD) }\end{array}$ & $\begin{array}{c}\text { Melt } \\
(\text { Mean }+ \\
\text { SD) }\end{array}$ \\
\hline $10^{\wedge} 7 \mathrm{k}^{\mathrm{a}}$ & 10.00 & 83.23 & $10.23,0.32$ & $83.30,0.1$ \\
$10^{\wedge} 7 \mathrm{k}$ & 10.45 & 83.37 & & \\
\hline $10^{\wedge} 6 \mathrm{k}$ & 13.15 & 83.57 & & \\
$10^{\wedge} 6 \mathrm{~s}^{\mathrm{b}}$ & 13.15 & 83.33 & $12.92,0.40$ & $83.51,0.16$ \\
$10^{\wedge} 6 \mathrm{c}^{\mathrm{c}}$ & 12.45 & 83.62 & & \\
\hline $10^{\wedge} 5 \mathrm{k}$ & 14.00 & 83.52 & & \\
$10^{\wedge} 5 \mathrm{~s}$ & 14.00 & 83.35 & $14.10,0.17$ & $83.48,0.11$ \\
$10^{\wedge} 5 \mathrm{c}$ & 14.30 & 83.57 & & \\
\hline $10^{\wedge} 4 \mathrm{k}$ & 15.45 & 83.56 & & \\
$10^{\wedge} 4 \mathrm{~s}$ & 16.45 & 83.33 & $16.30,0.78$ & $83.44,0.11$ \\
$10^{\wedge} 4 \mathrm{c}$ & 17.00 & 83.42 & & \\
\hline $10^{\wedge} 3 \mathrm{k}$ & 19.00 & 83.50 & & \\
$10^{\wedge} 3 \mathrm{~s}$ & 17.45 & 83.39 & $18.87,1.35$ & $83.45,0.06$ \\
$10^{\wedge} 3 \mathrm{c}$ & 20.15 & 83.47 & & \\
\hline $100 \mathrm{k}$ & 20.15 & 83.47 & & \\
$100 \mathrm{~s}$ & 18.45 & 83.09 & $20.35,2.00$ & $83.33,0.21$ \\
$100 \mathrm{c}$ & 22.45 & 83.42 & & \\
\hline $10 \mathrm{k}$ & 22.30 & 83.52 & & \\
$10 \mathrm{~s}$ & 21.00 & 83.42 & $22.43,1.50$ & $83.42,0.1$ \\
$10 \mathrm{c}$ & 24.00 & 83.33 & & \\
\hline $1 \mathrm{k}$ & 23.15 & 83.52 & & \\
$1 \mathrm{~s}$ & 22.30 & 83.42 & $23.92,2.11$ & $83.41,0.12$ \\
$1 \mathrm{c}$ & 26.30 & 83.28 & & \\
\hline $0.1 \mathrm{k}$ & 36.00 & 83.41 & & \\
$0.1 \mathrm{~s}$ & $-\mathrm{d}$ & 83.43 & $34.65,1.91$ & $83.39,0.06$ \\
$0.1 \mathrm{c}$ & 33.30 & 83.33 & & \\
\hline
\end{tabular}

2

3

4

5

6

7

\footnotetext{
\# The assay was performed in Genie III Real-time fluorometer, with the amplification times and annealing temperatures recorded at the end of each run. The samples were tested in different runs; *Template was serially diluted C. pecorum gDNA which genome copy number was determined by qPCR; from ${ }^{\text {a: }}$ koala Marsbar isolate; ${ }^{\text {b: }}$ sheep IPA isolate; and c: cattle E58 isolate; ${ }^{\mathrm{d}}$ : No amplification, but melt and annealing curve recorded.
} 


\section{Table 4(on next page)}

Reproducibility of the LAMP testing using clinical and cultured samples.

^The assay was performed in Genie III Real-time fluorometer, with the amplification times and annealing temperatures recorded at the end of each run. The samples were tested in three different runs by two different operators. 
1 Table 4. Reproducibility of the LAMP testing using clinical and cultured samples.

\begin{tabular}{|c|c|c|c|c|c|}
\hline Samples & $\operatorname{Run}^{\wedge}$ & $\begin{array}{c}\text { Time to } \\
\text { amplify (min) }\end{array}$ & $\begin{array}{l}\text { Melt } \\
\left({ }^{\circ} \mathrm{C}\right)\end{array}$ & $\begin{array}{c}\text { Time } \\
(\text { Mean }+ \text { SD) }\end{array}$ & $\begin{array}{c}\text { Melt } \\
(\text { Mean }+ \text { SD })\end{array}$ \\
\hline \multicolumn{6}{|c|}{ C. pecorum positive samples } \\
\hline \multirow{3}{*}{$\begin{array}{c}\text { Koala rectal } \\
\text { swab }\end{array}$} & 1 & 20.15 & 83.44 & & \\
\hline & 2 & 20.30 & 83.37 & $20.53,0.54$ & $83.32,0.16$ \\
\hline & 3 & 21.15 & 83.14 & & \\
\hline \multirow{3}{*}{ Marsbar DNA } & 1 & 13.50 & 83.50 & & \\
\hline & 2 & 13.15 & 83.52 & $13.27,0.20$ & $83.55,0.06$ \\
\hline & 3 & 13.15 & 83.62 & & \\
\hline \multirow{3}{*}{ Koala A2 DNA } & 1 & 12.00 & 83.35 & & \\
\hline & 2 & 11.00 & 83.45 & $11.43,0.51$ & $83.41,0.05$ \\
\hline & 3 & 11.30 & 83.43 & & \\
\hline \multirow{3}{*}{$\begin{array}{c}\text { RI koala UGT } \\
\text { swab }\end{array}$} & 1 & 17.00 & 83.34 & & \\
\hline & 2 & 18.00 & 83.21 & $17.72,0.62$ & $83.21,0.12$ \\
\hline & 3 & 18.15 & 83.09 & & \\
\hline \multirow{3}{*}{ L14 DNA } & 1 & 13.15 & 83.53 & & \\
\hline & 2 & 13.15 & 83.50 & $13.15,0$ & $83.50,0.02$ \\
\hline & 3 & 13.15 & 83.48 & & \\
\hline \multirow{3}{*}{ HsLuRz DNA } & 1 & 13.45 & 83.49 & & \\
\hline & 2 & 13.45 & 83.36 & $13.63,0.32$ & $83.40,0.08$ \\
\hline & 3 & 14.00 & 83.34 & & \\
\hline \multirow{3}{*}{$\begin{array}{l}\text { K20 cloaca } \\
\text { swab }\end{array}$} & 1 & 22.00 & 82.83 & & \\
\hline & 2 & 22.15 & 83.00 & $22.2,0.23$ & $83.01,0.19$ \\
\hline & 3 & 22.45 & 83.20 & & \\
\hline \multicolumn{6}{|c|}{ C. psittaci positive samples } \\
\hline \multirow{3}{*}{ Cr009 DNA } & 1 & 6.45 & 84.30 & & \\
\hline & 2 & 6.45 & 84.36 & $6.40,0.09$ & $84.33,0.03$ \\
\hline & 3 & 6.30 & 84.34 & & \\
\hline \multirow{3}{*}{ HoRE DNA } & 1 & 5.00 & 84.46 & & \\
\hline & 2 & 5.15 & 84.58 & $5.10,0.08$ & $84.45,0.14$ \\
\hline & 3 & 5.15 & 84.30 & & \\
\hline \multirow{3}{*}{ B2 DNA } & 1 & 10.30 & 84.08 & & \\
\hline & 2 & 10.00 & 84.20 & $10.10,0.17$ & $84.17,0.08$ \\
\hline & 3 & 10.00 & 84.24 & & \\
\hline \multirow{3}{*}{$\begin{array}{l}\text { Horse placental } \\
\text { swab }\end{array}$} & 1 & 11.15 & 82.90 & & \\
\hline & 2 & 10.30 & 83.42 & $10.58,0.49$ & $83.19,0.26$ \\
\hline & 3 & 10.30 & 83.24 & & \\
\hline \multirow{3}{*}{ Horse_pl DNA } & 1 & 10.30 & 84.53 & & \\
\hline & 2 & 12.00 & 84.21 & $10.87,0.98$ & $84.41,0.18$ \\
\hline & 3 & 10.30 & 84.50 & & \\
\hline
\end{tabular}


$2 \wedge$ The assay was performed in Genie III Real-time fluorometer, with the amplification times and annealing

3 temperatures recorded at the end of each run. The samples were tested in three different runs by two

4 different operators. 


\section{Table 5 (on next page)}

Comparison of the $C$. psittaci LAMP and qPCR methods for the organism detection in clinical samples. 
1 Table 5. Comparison of the $C$. psittaci LAMP and qPCR methods for the organism

2 detection in clinical samples.

\begin{tabular}{cccc}
\hline Test & qPCR +ve & qPCR -ve & qPCR Total \\
\hline LAMP +ve & 11 & 1 & 12 \\
\hline LAMP -ve & 1 & 13 & 14 \\
\hline LAMP Total & 12 & 14 & 26 \\
\hline
\end{tabular}

3 
Table 6 (on next page)

Comparison of the $C$. pecorum LAMP and qPCR methods for the organism detection in clinical samples. 
1 Table 6. Comparison of the $C$. pecorum LAMP and qPCR methods for the organism

2 detection in clinical samples.

\begin{tabular}{cccc}
\hline Test & $\mathbf{1 6 s}+\mathbf{v e}$ & $\mathbf{1 6 s}-\mathbf{v e}$ & 16 s Total \\
\hline LAMP +ve & 29 & 7 & 36 \\
\hline LAMP -ve & 3 & 24 & 27 \\
\hline LAMP Total & 32 & 31 & 63 \\
\hline
\end{tabular}

3 
Table 7 (on next page)

Comparison of $C$. pecorum LAMP and qPCR for organism detection using rapidly processed swab samples and their DNA extracts. 
Table 7. Comparison of $C$. pecorum LAMP and qPCR for organism detection using rapidly processed swab samples and their DNA extracts.

\begin{tabular}{|c|c|c|c|c|c|c|c|c|}
\hline Sample & $\begin{array}{l}\text { LAMPa }^{a} \text { result } \\
\text { for swab } \\
\text { suspension }\end{array}$ & $\begin{array}{c}\mathbf{q P C R}^{\mathrm{b}} \\
\text { result for } \\
\text { swab } \\
\text { suspension } \\
\end{array}$ & $\begin{array}{c}\text { LAMP result } \\
\text { for DNA } \\
\text { extract }\end{array}$ & $\begin{array}{l}\text { qPCR result for } \\
\text { DNA extract }\end{array}$ & $\begin{array}{l}\text { LAMP result } \\
\text { for "spiked" } \\
\text { swab } \\
\text { suspension } \\
\end{array}$ & $\begin{array}{c}\text { LAMP } \\
\text { result for } \\
\text { "spiked" } \\
\text { DNA extract } \\
\end{array}$ & $\begin{array}{l}\text { qPCR result for } \\
\text { "spiked" swab } \\
\text { suspension }\end{array}$ & $\begin{array}{l}\text { qPCR result for } \\
\text { "spiked" DNA } \\
\text { extract }\end{array}$ \\
\hline K1 ocular* & NEG & NEG & $0.00 / 83.49$ & NEG & NEG & - & NEG & - \\
\hline K6 ocular* & NEG & NEG & $21.00 / 83.23$ & $3 \times 10^{\wedge} 3(\mathrm{Ct} 20)$ & NEG & - & NEG & - \\
\hline K9 ocular* & NEG & NEG & $25.45 / 83.39$ & $287(\mathrm{Ct} 24)$ & NEG & - & NEG & - \\
\hline K2 ocular* & NEG & NEG & NEG & NEG & NEG & - & NEG & - \\
\hline R1 eye & $25.45 / 83.39$ & $222(\mathrm{Ct} 25)$ & $20.15 / 83.27$ & $750(\mathrm{Ct} 24)$ & - & - & - & - \\
\hline R1 cloaca & $30.00 / 83.34$ & NEG & NEG & NEG & $11.15 / 83.47$ & $12.15 / 83.42$ & $5 \times 10^{\wedge} 3(\mathrm{Ct} 17)$ & $1.5 \times 10^{\wedge} 3(\mathrm{Ct} 18)$ \\
\hline K eye & $27.00 / 83.15$ & NEG & $0.00 / 83.35$ & NEG & - & - & - & - \\
\hline Koala 2 eye & NEG & NEG & NEG & NEG & $11.00 / 83.51$ & $11.00 / 83.40$ & $1.2 \times 10^{\wedge} 3(\mathrm{Ct} 19)$ & $1.1 \times 10^{\wedge} 4(\mathrm{Ct} 15)$ \\
\hline Koala 2 cloaca & $27.30 / 83.77$ & $116(\mathrm{Ct} 26)$ & $21.30 / 83.49$ & $375(\mathrm{Ct} 25)$ & - & - & - & - \\
\hline Will Cloaca & $0.00 / 83.77$ & NEG & NEG & NEG & $12.00 / 83.45$ & $11.00 / 83.34$ & $1.5 \times 10^{\wedge} 3(\mathrm{Ct} 19)$ & $8 \times 10^{\wedge} 3(\mathrm{Ct} 17)$ \\
\hline 23117 Eye & $21.30 / 83.20$ & NEG & $23.15 / 83.23$ & $150(\mathrm{Ct} 25)$ & - & - & - & - \\
\hline 23117 Cloaca & $22.00 / 83.29$ & NEG & $24.00 / 83.15$ & $90(\mathrm{Ct} 27)$ & - & - & - & - \\
\hline Flyn eye & NEG & NEG & NEG & NEG & $12.30 / 83.50$ & $11.00 / 83.35$ & $1.9 \times 10^{\wedge} 3(\mathrm{Ct} 18)$ & $8.3 \times 10^{\wedge} 3(\mathrm{Ct} 16)$ \\
\hline Tyke eye & NEG & NEG & NEG & NEG & $12.00 / 83.44$ & $10.45 / 83.40$ & $1.3 \times 10^{\wedge} 3(\mathrm{Ct} 19)$ & $9 \times 10^{\wedge} 3(\mathrm{Ct} 16)$ \\
\hline Bill eye & NEG & NEG & NEG & NEG & $12.15 / 83.49$ & $10.45 / 83.34$ & $1.2 \times 10^{\wedge} 3(\mathrm{Ct} 19)$ & $1 \times 10^{\wedge} 4(\mathrm{Ct} 15)$ \\
\hline Ray eye & NEG & NEG & NEG & NEG & $12.45 / 83.49$ & $11.00 / 83.40$ & $4.7 \times 10^{\wedge} 3(\mathrm{Ct} 17)$ & $1 \times 10^{\wedge} 4(\mathrm{Ct} 15)$ \\
\hline Ray cloaca & NEG & NEG & NEG & NEG & $12.15 / 83.43$ & $11.00 / 83.30$ & $700(\mathrm{Ct} 20)$ & $9 \times 10^{\wedge} 3(\mathrm{Ct} 16)$ \\
\hline Koala F Eye & NEG & NEG & NEG & NEG & $11.45 / 83.45$ & $11.00 / 83.35$ & $1.3 \times 10^{\wedge} 3(\mathrm{Ct} 19)$ & $1.1 \times 10^{\wedge} 4(\mathrm{Ct} 15)$ \\
\hline
\end{tabular}

